\title{
Solar UV Spectral Irradiance Variations
}

\author{
Richard F. DONNELLY \\ Space Environment Lab., NOAA ERL Boulder, Colorado 80303, U.S.A.
}

(Received September 3, 1990; Revised October 29, 1990)

\begin{abstract}
Recent measurements of the relative temporal variations of solar UV radiation are reviewed. The long-term increase in solar UV irradiance during the rise of solar cycle 22 through April 1990 is comparable to the decrease from the solar cycle 21 peak in late 1981 to the 1986 minimum. Although several marked differences occur in the short-term or solar-rotational variations of the UV and Ottawa $10.7 \mathrm{~cm}$ radio flux (F10), the shape of the long-term solar-cycle increase of F10 is quite similar to that of UV enhancements.
\end{abstract}

\section{Introduction}

Solar ultraviolet (UV) radiation is important because it heats the stratosphere and photodissociates the major molecular constituents. This photodissociation leads to the formation of ozone and other important stratospheric constituents. Solar UV flux variations also contribute between one fifth and one third of the solar cycle enhancement of the total solar irradiance (LEAN, 1989; LONDON et al., 1989). It is therefore important to know both the absolute solar UV flux and its relative temporal variation as a function of wavelength. This paper discusses relative UV temporal variations; recent advances in determining the absolute flux are discussed elsewhere (LABS et al., 1987; NICOLET, 1989; VANHOOSIER et al., 1988).

The core-to-wing ratio [R(MgIIc/w, t)] of the $\mathrm{Mg}$ II h \& $\mathrm{k}$ absorption lines near $280 \mathrm{~nm}$ was defined by HEATH and SCHLESINGER (1986) for the Solar Backscatter Ultraviolet (SBUV) observations from the NIMBUS7 satellite. This ratio provides a relative photometry measure of solar UV variability that is insensitive to drifts in the instrument's transfer function. The present paper includes similar Mg II core-to-wing ratios for the rise of solar cycle 22 observed by the SBUV2 monitor on the NOAA9 satellite. HEATH and SCHLESINGER (1986) showed that $\mathrm{R}(\mathrm{MgIIc} / \mathrm{w}, \mathrm{t})$ can be used with linear regression equations to estimate the UV flux changes within the $175-290 \mathrm{~nm}$ wavelength range. The relative temporal fluctuations have been observed to be highly uniform in shape for short-term solar-rotational variations at wavelengths within the $175-290 \mathrm{~nm}$ range, although the percentage amplitude of these UV variations are a complicated function of wavelength (DONNELLY, 1988). This uniformity is caused by the UV radiation within that wavelength range being from essentially the same solar temperature range, namely from the upper photosphere, temperature-minimum region and chromosphere. Therefore, this uniformity is assumed to apply to long-term temporal changes as well as to short-term oscillations. $\mathrm{R}(\mathrm{MgIIc} / \mathrm{w}, \mathrm{t})$ is used here to represent the relative temporal variations of the solar UV flux in the 175-290 nm range. Long-term 
variations of the H Lyman alpha line measured by the SME satellite (ROTTMAN, 1988; BARTH et al., 1990) are also briefly discussed.

\section{Solar Cycle 22}

Figure 1 illustrates the temporal changes of solar UV flux as measured daily by the $\mathrm{Mg}$ II core-to-wing ratio from solar cycle minimum in 1986 through the fast rise to near the maximum of cycle 22 . The narrow peaks are caused by the solar rotation of active regions distributed inhomogeneously as a function of solar longitude. The peaks in late 1986 were produced by the first strong active regions of the new cycle. The 27-day solar rotational variations in 1989 and 1990 in Fig. 1 are quite large relative to the long-term increase from the solar cycle minimum, i.e. more than half the cycle's minimum to maximum in daily values and about five-sixths that in annually smoothed data. These large 27-day oscillations are produced by many active regions, often including several large groups in both the northern and southern solar hemispheres. In early 1988, an episode of two peaks per solar rotation occurred, which was caused by two peaks roughly $180^{\circ}$ apart in the solar longitudinal distribution of active regions. $\mathrm{R}(\mathrm{MgII} / \mathrm{w}, \mathrm{t})$ slightly under-represents the 13-day periodicity observed at most wavelengths in the $175-290 \mathrm{~nm}$ range. This is caused by the emission from an active region not decreasing to as low a value at the solar limb relative to that at the central meridian for the chromospheric $\mathrm{Mg}$ II $\mathrm{h} \& \mathrm{k}$ lines as it does for the more photospheric UV fluxes. During episodes of two peaks per rotation, the amplitude of the full-disk UV flux oscillations are never as strong as the large 27-day variations seen in 1989 and 1990. This weakness in 13-day UV amplitudes is partly due to the nonzero UV emission level of active regions at the limb. This weakness is also partly due to the episodes of two peaks roughly $180^{\circ}$ apart in the solar longitude having a combination of fewer and weaker active regions than in the cases of one strong peak per rotation. This latter effect is evident in the full-disk Ca-K plage index.

The data gap in late 1988 in Fig. 1 was caused by satellite orientation problems that have been avoided in subsequent years. For the previous solar cycle, 365-day running averages of $\mathrm{R}(\mathrm{MgII} / \mathrm{w}, \mathrm{t})$ peaked in 1981, about two years after the maximum value of the 13-month smoothed sunspot number. The $\mathrm{Mg}$ II ratio may therefore not yet have reached its

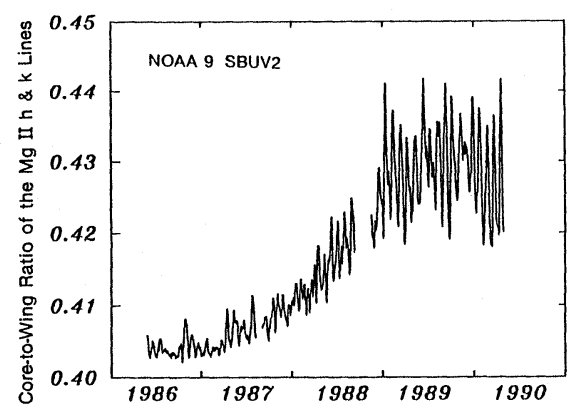

Fig. 1. Solar ultraviolet temporal variations during solar cycle 22 . 
highest 365-day average value for cycle 22.

The curve of daily NOAA9 core-to-wing ratios for the Mg II lines in Fig. 1 involves a ratio that has been modified with respect to the ratio used by HEATH and SCHLESINGER (1986) for NIMBUS7. The wavelengths of the flux measurements in both wings were changed to avoid instrumental noise and drift problems associated with one of the three intensity ranges used to cover the full dynamic range of the NOAA9 SBUV2 instrument. This modification causes Mg II ratios in Fig. 1 to be more than 50\% higher in 1986 than reported by HEATH and SCHLESINGER (1986) for the same days; however, the relative temporal variations of the Mg II ratio from concurrent NIMBUS7 and NOAA9 observations have the same temporal shape.

\section{Comparison with Cycle 21}

The bottom graph in Fig. 2 shows the combined NOAA9 and NIMBUS7 Mg II ratios obtained by converting the NOAA9 modified ratios in Fig. 1 to the equivalent NIMBUS7 ratios using a linear regression relation. Note that the peak Mg II ratios in Fig. 2 in 1989 and 1990 during solar cycle 22 are comparable to the peak ratios in 1979-1981 during solar cycle 21. For 365-day running averages, the maximum value of $\mathrm{R}(\mathrm{MgIIc} / \mathrm{w}, \mathrm{t})$ in 1989 is nearly identical to that for cycle 21 in 1981. The large UV solar rotational variations in 1989 through April 1990 are comparable in magnitude to those in late 1979, mid 1980 and mid 1982. Figure 2 shows intermediate-term oscillations in the $\mathrm{Mg}$ II ratio with periods near half a year are present from late 1979 to mid 1981 near the peak of cycle 21 and others with periods slightly less than a year are present in 1982-1984 during the decay of cycle 21. Intermediate-term changes in $\mathrm{R}(\mathrm{MgIIc} / \mathrm{w}, \mathrm{t})$ of magnitude comparable to those in 1979-1984 are not evident in Fig. 2 in 1987-1988 during the rise of cycle 22.

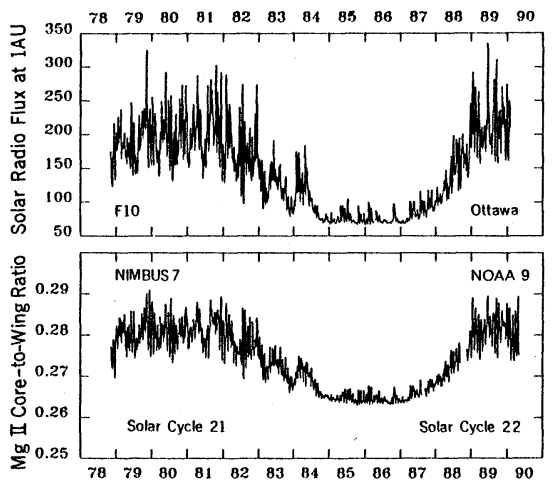

Fig. 2. Temporal variations of daily values of the solar ultraviolet and $10.7 \mathrm{~cm}$ flux from the peak of solar cycle 21 through the rise of cycle 22 . 


\section{Comparison with F10}

Identifying the similarities and differences between $\mathrm{F} 10$ and $\mathrm{R}(\mathrm{MgIIc} / \mathrm{w}, \mathrm{t})$ is important because F10 may provide good estimates of $\mathrm{R}(\mathrm{MgIIc} / \mathrm{w}, \mathrm{t})$ back to 1947 . However, the shortterm UV variations have been shown (DONNELLY, 1987) to differ significantly from those of F10 in the following ways: (1) The average amplitude of the short-term (days, weeks) solarrotational changes is larger relative to the long-term minimum to maximum solar cycle enhancement for F10 than in the case of UV flux variations, which is evident in Fig. 2. (2) During an episode of strong 27-day solar rotational oscillations produced by a major group of active regions, F10 evolves more rapidly than the UV enhancements, i.e. F10 usually peaks on the first rotation and decreases more rapidly on subsequent rotations while the UV usually peaks on the second rotation and decays slowly for several more rotations. The large amplitude single-rotation peaks in F10 in Fig. 2 in November 1979, December 1982 and June 1989 are examples of this behavior. In contrast to F10, the Mg II ratio shows many more solar-rotational variations during 1985-1986 (Fig. 2). These extra variations provide evidence of the greater persistence in the UV enhancements than in F10. (3) UV fluctuations occasionally have episodes with two-peaks per rotation or 13-day periodicity, while F10 is devoid of 13-day periodicity. (4) The solar-rotational temporal half widths of F10 are larger time intervals than those for UV fluxes; consequently, the 27-day rotational valleys are wider and flatter in the UV than in F10.

These four differences in short-term variations are probably caused by F10 including coronal and transition region emission from active regions in addition to chromospheric thermal bremsstrahlung. The latter emission should be closely related to the photospheric and chromospheric UV emission of active regions. The $10.7 \mathrm{~cm}$ emission from the higher regions apparently is generated by thermal and nonthermal electrons, including both gyroresonance and bremsstrahlung emission (GELFREIKH, 1990; AKHMEDOV et al., 1986).

BARTH et al. (1990) found that the local minima per solar rotation form a nearly constant background level in F10 during 1985-1986, while the corresponding minima for the SME measurements of Lyman alpha decay slowly to mid 1986. A similar effect can be seen in Fig. 2 as a small curvature in the long-term variations of the local minima per solar rotation for the Mg II ratio in 1985-1986. Figure 3 shows scatter diagrams of daily values of
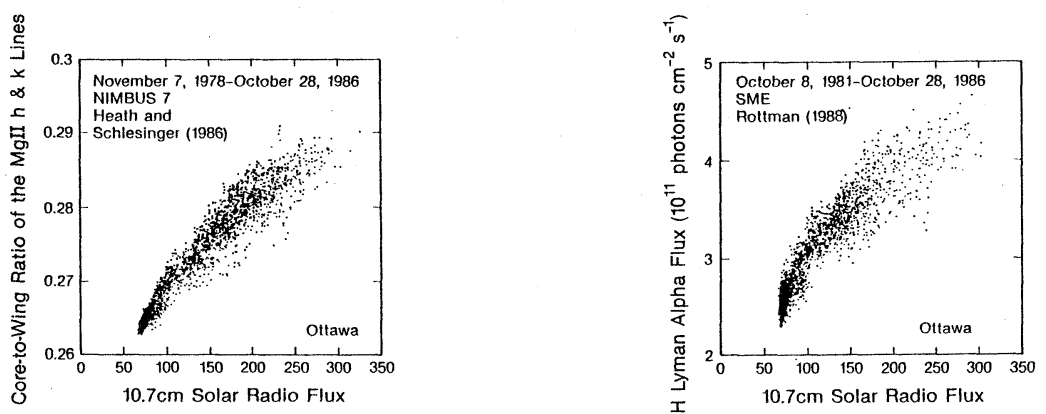

Fig. 3. Daily values of R(MgIIc/w, t) and H Lyman alpha flux versus F10. Note the nonlinear effect at low F10 values. 
the $\mathrm{Mg}$ II ratio versus F10 and Lyman alpha flux versus F10. The curve of rotational minima for $\mathrm{R}(\mathrm{MgIIc} / \mathrm{w}, \mathrm{t})$ found in Fig. 2 for 1985-1986 causes a slight curvature in chromospheric $\mathrm{R}(\mathrm{MgIIc} / \mathrm{w}, \mathrm{t})$ versus F10 at low intensities in Fig. 3. The larger curvature in Lyman alpha of BARTH et al. (1990) causes the pronounced deviation from a linear trend at low flux levels for Lyman alpha versus F10 in Fig. 3. The scatter diagram for ground-based measurements of the equivalent width of the He I line at 10830 A (HARVEY, 1984) versus F10 (not shown) is quite similar to that for Lyman alpha. The rotational minima for the coronal green line decay throughout 1985 to a minimum in September 1986 (RYBANSKY et al., 1988). These results for Mg II, Lyman alpha, He I $10830 \mathrm{~A}$ and the coronal green line form a progression with increasing nonlinearity or slower decay at low intensities in the relation of long-term variations with respect to F10 from lower to higher temperature source regions. Consequently, this nonlinearity is considered to be real and not caused by unknown drifts in the instrumentation.

The extreme outliers from a linear trend in Fig. 3 tend to fall below and to the right of the main trend. These outliers come from the first rotation of episodes of 27-day oscillations when F10 is much larger than the corresponding UV enhancement (DONNELLY, 1987). Those cases can also be seen in Fig. 2 as the large short-term peaks in F10 that are accompanied by small solar-rotational UV peaks, for example in November 1979. The fact that solar rotational variations during episodes of 27-day oscillations persist longer for UV fluxes than for F10 is quite evident from the more numerous peaks in the $\mathrm{Mg} \mathrm{II}$ ratio in Fig. 2 in 19851986, during the solar cycle minimum. These marked differences in short-term variations caused BARTH et al. (1990) to find variations from one solar rotation to the next in the linear regression relation between F10 and the solar Lyman alpha flux. These differences in shortterm variations are not random occurrences, but are systematically related to the short-term evolution of active regions and their remnants.

Figure 4 shows a linear relation between 365-day running averages of the $\mathrm{Mg}$ II ratio and F10 for the same data sets shown in Fig. 2. Note the slight offset between the decay of cycle 21 and the rise of cycle 22, which forms a narrow, closed hysteresis loop rather than a single straight line. The data near the peak of solar cycle 21, from mid 1978 through 1981, produce the short thick bar of points labeled A in Fig. 4. The late 1989 values close with cluster A from the maximum of cycle 21 . This closure is important because it shows the

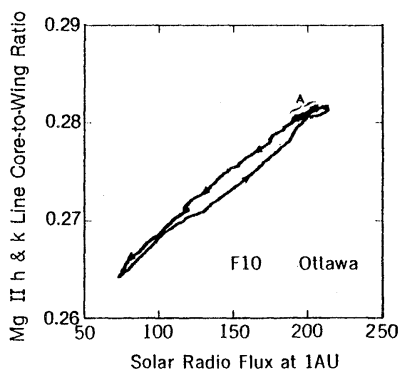

Fig. 4. Daily values of 365-day averages of $R(\mathrm{MgIIc} / \mathrm{w}, \mathrm{t})$ versus 365 -day averages of $\mathrm{F} 10$. The arrowheads point in the direction of increasing time. The thick short bar marked A comes from mid 1979 through 1981, the peak of cycle 21 . 
results for cycles 21 and 22 agree well when the long-term averages are nearly constant with time, as they are near the peaks of these two cycles. The differencès between the decay of cycle 21 and the rise of cycle 22 in Fig. 4 occur when the long-term averages are changing rapidly with time. The 1985-1986 values in Fig. 2 produce the bottom point in the loop in Fig. 4.

This hysteresis can be explained qualitatively by the solar activity changes in the 10.7 $\mathrm{cm}$ solar radio flux. F10 emission comes from chromospheric thermal bremsstrahlung and coronal and transition region emission from thermal and probably nonthermal electrons through bremsstrahlung emission and interactions with the strong magnetic fields of active regions (GELFREIKH, 1990). The chromospheric component of F10 should be closely related to the temporal variations of the chromospheric $\mathrm{Mg} \mathrm{II} \mathrm{h} \mathrm{\&} \mathrm{k}$ lines for both the short- and long-term variations. Since the persistence of short-term solar-rotational variations of F10 is much less than that for chromospheric UV and EUV emissions (DONNELLY, 1987), the coronal component of F10 must evolve faster than the chromospheric component and UV emission. Assume the solar cycle variations are simply the overlapping summed effects of the evolution (birth, growth, peak and decay) of many active regions and their remnants. Since F10 evolves more rapidly than the UV flux for each active region, then the sum over all regions seen from Earth and consequently the long-term average of the coronal component of F10 should rise and decay a little earlier than the chromospheric component. The two components should be well correlated simply because they involve the same active regions. The overall linearity in Fig. 4 comes then from the close relation of the chromospheric F10 emission with the UV flux combined with an approximate relation between the coronal and chromospheric components. The narrow hysteresis loop then comes from the 365-day average of the coronal component of F10 preceding that of the chromospheric component. Shifting the F10 data to 27 days after the day it was observed produces results like those in Fig. 4 except the hysteresis loop narrows to nearly one line.

Separating F10 into long and short-term variations improves the linear-regression estimate of UV fluxes. This approach accounts for the amplitude of the short-term solarrotational oscillations relative to the long-term solar cycle increase differing for F10 and the UV fluxes. Figure 5 illustrates the estimates of long-term variations of the $\mathrm{Mg}$ II ratio based on 81-day averages of F10. The 81-day averages of $\mathrm{Mg}$ II observations are included for comparison. These estimates are excellent for the long-term (years) UV variations but

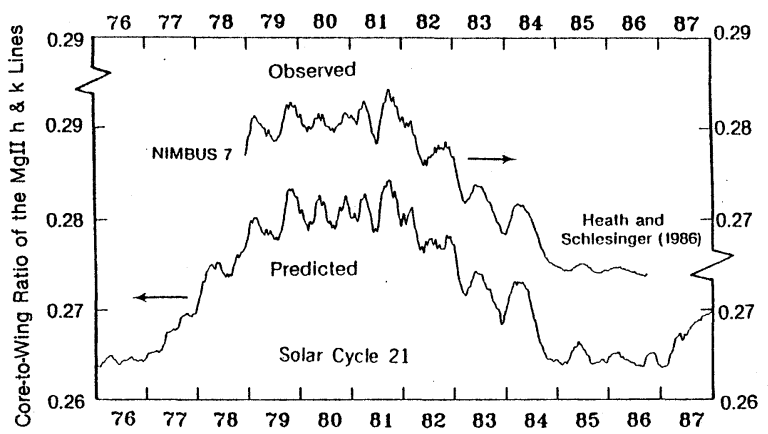

Fig. 5. Comparison of observed and predicted 81-day average $\mathrm{R}(\mathrm{MgIIc} / \mathrm{w})$. 
overestimate slightly the intermediate-term variations (months). This overestimation is caused by the amplitude of the intermediate-term enhancements in F10 being larger relative to the long-term solar cycle amplitude than in the case of UV fluxes. It can be reduced by using a longer averaging interval.

Figure 5 shows intermediate-term oscillations with periods near half a year during the years 1979-1981 and periods slightly less than a year during the decay of cycle 21 . These intermediate-term oscillations are smaller relative to the solar cycle variation and decay slower from each local maximum to the subsequent minimum in $\mathrm{R}(\mathrm{MgIIc} / \mathrm{w}, \mathrm{t})$ than in $\mathrm{F} 10$. Intermediate-term changes appear in F10 (Fig. 2) during the rise of cycle 22 and in the F10based predictions (Fig. 5) during the rise of cycle 21 . The fact they are not evident in the rise of the Mg II ratio in 1987-1988 in Fig. 2 probably results from their being slower and smaller in $\mathrm{R}(\mathrm{MgIIc} / \mathrm{w}, \mathrm{t})$ than in F10. This would tend to smooth out their effect in $\mathrm{R}(\mathrm{MgIIc} / \mathrm{w}, \mathrm{t})$ during the rapid rise of the cycle and make them less distinguishable.

Another technique for improving estimates of the $\mathrm{Mg}$ II ratio based on regression relations with F10 is to include the sunspot blocking function of HOYT and EDDY (1982) as a second regression variable. Their sunspot blocking function is a sum over the visible solar disk of all the sunspot umbral and penumbral areas weighted by a contrast function. Including the sunspot blocking function in the regression of daily values helps correct for the large F10 solar-rotation variations early in episodes of major groups of active regions. The sunspot blocking function is large on the first rotation of episodes of major groups of activity but is weak to negligible on later rotations. Mathematically, this correction is compensating for the more rapid evolution of F10 than for UV fluxes. Physically, this correction is probably roughly compensating for the gyro-resonance and nonthermal coronal emission in F10 associated with the strong magnetic fields from sunspots. An alternative physical interpretation for the improvement found using the sunspot blocking function is that the differences in the F10 time dependence relative to that of the UV flux is caused by thermal bremsstrahlung from the transition region and corona in closed magnetic flux tubes in active regions. In this second interpretation, the sunspot blocking function would provide an estimate of the number of strong-field tubes. The chromospheric bremsstrahlung component of F10 should be closely related to the $\mathrm{Mg}$ II ratio. Contrarily, soft X-rays and the coronal green line (RYBANSKI et al., 1988) have a much stronger long-term solar cycle peak in late 1981 relative to the late 1979 levels than in the case of either F10 or R(MgIIc/w, t). This alternative explanation would conflict with the solar cycle shape for these independent coronal data sets.

\section{Conclusions}

The daily and 365-day smoothed peak intensities in the Mg II core-to-wing ratio $\mathrm{R}(\mathrm{MgIIc} / \mathrm{w}, \mathrm{t})$ observed in solar cycle 22 through April 1990 are comparable to the peak values of solar cycle 21 . R(MgIIc/w, t) increased by about $10 \%$ in daily values and $7 \%$ in 365-day averages from 1986 to 1989. Separating F10 into short-term (days, weeks) and longterm (years) changes improves the fit to the $\mathrm{Mg}$ II ratio. The long-term relative temporal variations of $\mathrm{R}(\mathrm{MgIIc} / \mathrm{w}, \mathrm{t})$ and $\mathrm{F} 10$ are quite similar with F10 leading the $\mathrm{Mg}$ II ratio slightly, which is probably caused by the more rapidly evolving gyroresonance and nonthermal coronal component in F10. 


\section{REFERENCES}

Akhmedov, Sh. B., V. N. Borvik, G. B. Gelfreikh, V. M. Bogod, A. N. Korzhavin, Z. E. Petrov, V. N. DikiJ, K. R. LANG, and R. F. Willson, Structure of a Solar Active Region from RATAN 600 and Very Large Array Observations, Astrophys. J., 301, 460-464, 1986.

Barth, C. A., W. K. Tobiska, G. J. Rottman, and O. R. White, Comparison of $10.7 \mathrm{~cm}$ radio flux with SME solar lyman alpha flux, Geophys. Res. L., 17, 571-574, 1990.

Donnelly, R. F., Temporal trends of solar EUV and UV full-disk fluxes, Solar Phys., 109, 37-58, 1987.

Donnelly, R. F., Uniformity in solar UV flux variations important to the stratosphere, Annales Geophys., 6, 417-424, 1988.

GelfreikH, G. B., Inner Corona from Solar Radio Observations, to be published in Advances of Space Res., the Proc. of COSPAR Symp. 9 on Space Observations of the Solar Corona and Origin of the Solar Wind, held June 27-29, 1990, in The Hague, Netherlands, 1990.

HARVEY, J., Helium 10830A Irradiance: 1975-1983, in Solar Irradiance Variations on Active Region Time Scales, NASA Conference Publ. 2310, edited by B. J. LaBonte et al., 197-211, 1984.

HeAth, D. F. and B. M. Schlesinger, Mg 280-nm doublet as a monitor of changes in solar ultraviolet irradiance, J. Geophys. Res., 91, 8672-8682, 1986.

HoYT, D. V. and J. A. EDDY, An Atlas of Variations in the Solar Constant Caused by Sunspot Blocking and Facular Emissions from 1874 to 1981, 106 pp., NCAR Tech. Note 194+STR, NCAR, Boulder, Colorado, 1982.

Labs, D., H. Neckel, P. C. Simon, and G. Thuillier, Ultraviolet solar irradiance measurement from 200 to 358 nm during Spacelab 1 mission, Solar Phys., 107, 203-219, 1987.

LEAN, J., Contribution of ultraviolet irradiance variations to changes in the sun's total irradiance, Sci., 244, 197-200, 1989.

LONDON, J., J. PAP, and G. J. ROTTMAN, Observed solar near UV variability: a contribution to variations of the solar constant, Handbook for MAP, 29, 9-12, 1989.

Nicolet, M., Solar spectral irradiances with their diversity between 120 and $900 \mathrm{~nm}$, Planet. Space Sci., 37, 1249-1289, 1989.

Rottman, G. J., Observations of solar UV and EUV variability, Adv. Space Res., 8, (7)53-(7)66, 1988.

Rybanski, M., V. Rusin, and E. DzIFCAKova, Coronal index of solar activity, V. Years 1977-1986, Bull. Astron. Inst. Czechoslovakia, 39, 106-119, 1988.

VAnhoosier, M. E., J.-D. F. BARtoe, G. E. Brueckner, and D. K. Prinz, Absolute solar spectral irradiance $120 \mathrm{~nm}-400 \mathrm{~nm}$ (Results from the Solar Ultraviolet Spectral Irradiance Monitor - SUSIM experiment on board Spacelab 2), Astro. Lett.. \& Commun., 27, 163-168, 1988. 